\title{
Acknowledgment of Reviewers 2019
}

The editors and staff of the Journal of Women's Health are grateful to the many experts who have volunteered their time in 2019 to review submitted manuscripts. The ongoing efforts of our authors, reviewers, section editors, and editorial board members have helped the Journal of Women's Health to become the very best journal covering the full range of women's health across the lifespan. Thank you for all of your efforts on behalf of the Journal for its continued success! A list of our 2019 reviewers is available below.

Abdelmoneim, Sahar

Accortt, Eynav

Akinlotan, Marvellous

Alfhaily, Fadi

Allen, Lindsay

Allison, Kimberly H.

Allsworth, Jenifer

Amin-Esmaeili, Masoumeh

Amugsi, Dickson Abanimi

Amundsen, Cindy

Anderson, Chelsea

Ansa, Benjamin.

Antao, Vinicius

Anttila, A

Arndt, V.

Arnold, Ahran

Atkins, David

Averbach, Sarah

Ayres, Margaret E Rinehart

Azad, Nahid

Azrad, Maria

Babapour Mofrad, Rosha

Bachmann, Gloria

Badour, Christal

Baecher-Lind, Laura

Bahamondes, Luis

Baker, Jennifer

Baldwin, Maureen

Ballard, Rachel

Bankston, Karen

Bao, Yicheng

Baranoski, Amy

Barger, MK

Barker, Lucy

Barkin, Jennifer

Barlow, William

Barnes, Kylie

Baron, Kelly

Barraco, Robert

Bartha, Jose

Basili, Stefania

Basso, Olga

Battaglia, Tracy

Batur, Pelin

Baum, Marianna K.

Bavendam, Tamara
Bazi, Tony

Beaber, Elisabeth

Bednarczyk, Robert

Behbehani, Sadikah

Beiki, Omid

Belibasakis, Georgios

Bell, Shannon

Bellcross, CA

Bello, Jennifer

Ben Gal, Tuvia

Benagiano, G

Benetti-Pinto, Cristina

Bennett, Amanda

Bennett, Christina

Bennett, Wendy

Best, Jennifer

Bhasin, Prerna

Bhat, Amritha

Bickel, Janet

Billings, Deborah

Blacakard, Jason

Black, Katherine

Blakeslee, Sarah

Blauwet, Cheri

Boatright, Dowin

Bodurtha, Joann

Boghossian, Nansi S

Boiko, Julie

Bonomi, Amy

Bot, Mariska

Boulet, Sheree

Boutot, Maegan

Bowen, Deborah

Bracamonte-Baran, William

Brandt, Cynthia

Breathett, Khadijah

Brem, RF

Brennan, PA

Brittain, Kirsty

Brousseau, Erin

Brown, Amy

Brown, Heidi

Brown, Kara

Brunner Huber, Larissa

Buck, Gregory

Bullock, Linda
Burks, Heather

Buzi, Ruth

Calhoun, Elizabeth

Cancelo, MJ

Cancian, Madeline

Canda, MT

Cantor, Amy

Cantu, Adelita

Carapinha, René

Carnes, Molly

Carpenter, Shannon

Cartwright, Alice

Casimiro, Isabel

Castaño, Paula

Cecchini, Reena

Cesta, Carolyn

Chakrabarti, Subho

Chambliss, Linda R.

Chang, Yu-Chao

Chapman, Christina

Chen, Lee-may

Chernick, Lauren

Cheslack-Postava, K.

Choo, Esther

Chung, Doreen

Chung, Seockhoon

Cleland, John

Clements, Karen

Cochrane, Barbara

Cohen, Adi

Cohen, Rebecca

Cohen, Sarah

Coleman, Valerie

Cook, Mackenzie

Cottrell, Lesley

Coutinho, Larissa

Curtis, Kathryn

Cyrino, Renata

Daniels, Kimberly

Datta, Geetanjali

Dattijo, LM

Davids, Jennifer

Davis, Ann

Davisson, Laura

Daw, Jamie

Dawson Rose, Carol 
Day, Carolyn

De Genna, Natacha

de Jonge, Lilian

De Rosa, Salvatore

de Schepper, EI

Deans, Elizabeth

Demirci, Jill

DeSisto, Carla

DeVon, Holli

Di Giuseppe, Gabriella

Dichter, ME

Dillon, PM

Dimai, Hans Peter

Dimbuene, Zacharie

Diniz da Rosa, Marine Raquel

Dmochowski, Roger

Dobrosielski, Devon

Dodson, Nancy

Dolbier, C.

Dorfman, Caroline

D'Oria, Robyn

Downs, Robert

Drake, Isabel

Duncan, Meredith

Durand, Madeleine

Dyer, Karen

Dzierzewski, Joseph

Eastwood, Jo-Ann

Ebell, Mark

Eden, Karen B.

Edwards, Rodney

Ellinas, Elizabeth

Emerson, Amanda

Englander, Meridith

Erekson, Elisabeth

Erekson, Elisabeth

Evans, Megan

Evans, Susan

Everett, Bethany

Fairweather, DeLisa

Falasinnu, Titilola

Falcone, T.

Fall-Dickson, Jane

Farid, Huma

Farmer, Melissa

Farrell, Stephen

Farukhi, Zareen

Fedeles, Flavia

Feldon, David

Ferrero, Simone

Figueroa, Chantal

Findley, Molly

Fisher, William

Flatt, Jason

Flynn, Heather

Fok, Cynthia

Folger, Alonzo

Folger, Suzanne

Folmar, Steven J.

Forney, Larry

Freeman, Marlene

Freeman, Ruth
Frew, Julia

Friedrich, Patricia

Fritel, Xavier

Fryer, Kimberly

Fuster, Daniel

Garcia, Edgard

Garland, Sheryl

Garner, Christine

Garza, Mary

Geller, Stacie

Gemmill, Alison

Gibbs, Susannah

Gill, Ranjodh

Glasser, Saralee

Glazer, Kim

Glover, Angelica

Gold, Jessica

Goldstein, Jill

Goldstein, Lizabeth

Gondwe, Tamala

Goodman, Suzan

Gotlieb, Walter

Gottlieb, Barbara

Gould, Neda

Gregory, Emily

Gremigni, Paola

Grindlay, K.

Gross, Amy

Groth, SW

Groves, Allison

Gunn, Christine

Gutman, Colleen

Haas, David

Hacker, Karen

Hacker, Michele

Hackley, Barbara

Hagobian, Todd

Hamilton, Erin

Hampton, RM

Handberg, Eileen

Hanscom, Brett

Hare, Joshua

Harvey, Megan

Haselschwerdt, Megan

Haskell, Sally

Hastie, Claire

Hatch, Elizabeth

Hauspurg-Janicki, Alisse

Hay-Smith, J.

Heberlein, Emily

Heikinheimo, Oskari

Hein, Laura

Hernandez, Angela

Herrera-Escobar, Juan Pablo

Hess, Kristen

Hewitt, Belinda

Higgins, Stephen

Hipwell, Alison

Hirsch, Oliver

Hoare, Derek

Hogue, Carol

Honda, Tomoko
Hopkins, Kristine

Horner-Johnson, Willi

Housten, Ashley

Howell, EM

Howell, Lydia

Huang, Yongmei

Huffstetler, Alison

Hux, Vanessa

Hyun, Karice

Ihongbe, Timothy

Ioannidou, Effie

Isaacs, Christine

Isiadinso, Ijeoma

Ismaili M'hamdi, Hafez

Isono, Wataru

Jackson, Katherine

Jacobs, Jr., David

Janicki Deverts, Denise

Jaspers, Ilona

Javed, Fawad

Jayasekera, Jinani

Jeffe, Donna

Johnson, Lee Ann

Johnson, Timothy

Kaatz, Anna

Kalejta, Robert

Kaminsky, Leonard

Kanic, Vojko

Kapp, Julie M.

Kato, Ken

Katon, Jodie

Kawaguchi, Yoshiharu

Kellesarian, Sergio

Kessels, Sharon

Khair, Kate

Khullar, Dhruv

Kilpatrick, Sarah

Kilpi, Fanny

Kingsberg, Sheryl

Kinsky, Suzanne

Kirby, Russell

Kirca, Nurcan

Kirchengast, S.

Kirkegaard, Helene

Klaritsch, Philipp

Kling, Juliana

Koch, Abigail

Kolehmainen, Christine

Korevaar, Tim

Kostev, Karel

Kouyoumdjian, Fiona

Kovacevic, Maja

Koyama, Atsuko

Kroelinger, Charlan

Krukowski, Rebecca

Kurti, Allison

Kusche-Vihrog, Kristina

Kusunoki, Yasamin

Lagerlund, Magdalena

Lall, Michelle

Lane-Cordova, Abbi

Laufer Perl, Michal 
Laukkanen, Jari

Lavie, Carl

Lawrence, J.

Leddy, Meaghan

Ledoux, Tracey

Lee, Ajin

Lee, You-Geon

Lee-Felker, Stephanie

Lenze, Shannon

Levine, Rachel

Leyser-Whalen, Ophra

Liddon, NC

Liebergall, Michal

Lira Júnior, Ronaldo

Liu, Can

Liu, Lihua

Løkkegaard, Ellen

Looby, Sara

López, Rocio

Lòpez-De Fede, Ana

López-Soto, Pablo Jesús

Loucks, Tammy

Loue, Sana

Ludovisi, M.

Lundberg, Gina

Lust, Katherine

Lynch, Catherine

Lynch, Molly

Ma, Grace

Maas, Angela

MacGregor, Andrew

Mack, Wendy

Madigan, David

Magalhaes, Thais

Maggioni, Aldo

Magnus, Per

Mahapatra, Saswati

Makari-Judson, Grace

Malm, Heli

Mamas, Mamas

Maness, Sarah

Manhart, Lisa

Mansoor, Hend

Mao, Constance

Marcelin, Jasmine

Marshall, Ariela

Marshall, Lynn

Martinez, Gladys

Mathews, Lena

Mayer, Stephanie

Mazure, Carolyn

McCall-Hosenfield, Jennifer

Mcclintock, Adelaide

McClish, Donna

McClurg, Doreen

McCubbin, Andrea

McDermott, Mollie

McDonald, Jill

McDowell, Joanne

McDowell, Michal

McGhee, Sarah

McGill, Janet
McGregor, Alyson

McLemore, Monica

McNeill, Diana

Medina-Inojosa, Jose

Mehta, Ambereen

Mehta, Pooja

Melloni, Chiara

Merritt, Victoria

Merz, C. Noel

Meschke, Laurie

Metge, CJ

Meyer, Jaimie

Michos, Erin

Micussi, Thereza

Miller, Carrie

Miller, Jacqueline

Miller, Melissa

Miller, Virginia M.

Minhas, Anum

Minissian, Margo

Missmer, Stacey

Mitchell, Helene

Mitchell, Jason

Mitro, Susanna

Mochari-Greenberger, Heidi

Mollen, Cynthia

Moreau, Jessica

Moreno, $\mathrm{R}$.

Morgan, Helen

Morgan, Isabel

Morosky, Christopher

Morse, DS

Mulvagh, Sharon

Murphey, Christina

Murray, Melanie

Myers, Sharon

Myles, Ranell

Narod, Steven

Nelson, Anita

Nestler, John

Newson, Louise

Newton, Edward

Niehaus, William

Nitschmann, Caroline

Nittrouer, Christine

Norwood, Carolette

Nosek, Margaret

Nsuami, M. Jacques

Oertelt-Prigione, Sabine

O'Hara, Michael W.

Ohlendorf, Jennifer

Ondersma, Steven

O’Neill, Suzanne C.

Onken, Cheryl

Oparil, Suzanne

Osborne, Vicki

O'Toole, B.

Oyebode, Oluwafemi

Palacios, Santiago

Papademetriou, Vasilios

Parekh, Ranna

Parise, CA
Pariser, Anne

Parish, Sharon

Park, Chan Jin

Parker, Melissa

Peipert, Jeff

Pendergast, Julie

Pérez, Ashley

Perman, Sarah

Peterman, Jim

Peters, Ellen

Petersen, Emily

Peterson, Brennan

Philipsen, Maike

Phillips-Howard, Penelope

Pinkerton, JoAnn

Pinter, Bojana

Pluskiewicz, Wojciech

Poleshuck, E.

Popova, Polina

Post, Kathryn

Potter, JE

Powe, Camille

Prather, Cynthia

Pregler, Janet

Priest, $\mathrm{P}$

Prieto-Sánchez, MTeresa

Prince, Louise

Pritts, Elizabeth

Qian, Jingjing

Quinn, Deirdre

Racette, Susan

Radford, Diane

Rahm, Alanna K.

Rahman, Belinda

Rama, Jennifer

Raymond, Nancy

Reame, Nancy

Reese, Jennifer

Reneker, Jennifer

Reynolds, Harmony

Reynolds, Monica

Rhoten, Bethany

Ricci, Maria Teresa

Ripley, Betsy

Robbins, Cheryl

Roberts, Andrea

Rojek, Mary

Rom, Oren

Rominski, Sarah

Rondon, Marta

Ross, Sue

Rosser, Mary

Rouen, Patricia

Ruben, Mollie

Rufino, Andrea Cronemberger

Ryan, Ginny

Sadler, Anne

Saglam, Ebru

Sahley, Christie

Sahni, Ashima

Salahuddin, Meliha

Samari, Goleen 
Sanderlin, Ashley

Sandstrom, Gillian

Santoro, Luca

Savageau, JA

Scantlebury, Dawn

Scarinci, Isabel

Schellinger, Megan

Schonfeld, Sara

Schrager, Sarina

Schreiber, Shaul

Schwarz, Bimla

Scranton, Alexandra

Sederholm Lawesson, Sofia

Seifer, David

Selk, Sabrina

Shah, Brandi

Sharara, Fady

Sharma, Ritu

Shatzer, John

Sheehan, Connor

Shellhaas, Cynthia

Shepherd-Banigan, Megan

Sheth, AN

Shillcutt, Sasha

Shimada, Stephanie

Sidebottom, Abbey

Silver, Julie

Silverstein, Brett

Sima, Adam

Siminoff, Laura

Simonetti, Joseph

Singhal, Divya

Skurnik, Geraldine

Smeltzer, Suzanne

Smith, BN

Smith, Peggy

Smith, Tyler

Solomon, Susan

Sommer, Marni

Spector, Nancy

Sperlich, Mickey

Spernal, Suzanne

Sriraman, Natasha

Stanczyk, Frank

Stark, A.
Stayner, Leslie

Steenland, Maria

Steiner, Riley

Sterling, Evelina

Stevens, Gregory

Stewart, Donna

Stewart, Sherri

Stockman, Jamila

Stöllberger, Claudia

Stroud, Laura

Stuart, Jennifer

Stulberg, Debra

Subasinghe, Asvini

Sufrin, Carolyn

Sullivan, Tami

Suter, Melissa Anne

Sweeney, Lori

Tabaac, Ari

Tambalis, Kostas

Taplin, Stephen

Taubenberger, Simone

Teal, Stephanie

Templeton, Kimberly

Teslyar, Polina

Thomas, Holly

Thorndyke, Luanne

Tolcher, Mary Catherine

Tolley, Elizabeth

Tom, Sarah

Tsai, Pei-Shan

Tsai, Sandra

Tsigas, Eleni

Tsui, Amy

Tucker Edmonds, Brownsyne

Turvey, Carolyn

Upadhyay, Ushma

Upson, Kristen

Vaid, Isam

Valenstein, Marcia

Valentine, Rudy

van Kemenade, F.

Vanderpool, Robin

Veldhuizen, Scott

Vermeulen, KM

Vernon, Marlo
Viglianti, Elizabeth

Vogel, VG

von Birgelen, Clemens

von Kaisenberg, Constantin

Voss, Joachim

Walker, Shawn

Wall, Kristin

Waller, J.

Walsh, Mary Norine

Wandell, Per

Wang, Jeffrey C.

Wang, Shi-Heng

Warembourg, Charline

Waring, Molly

Weil, Amy

Weinmann, Sheila

Wen, Shi-Wu

Wenzel, Thomas

Werbinski, Jan

Werbinski, Janice

White, Kari

White, Mary

Wilkinson, Tracey

Williams-Brown, Cynthia

Witkin, Steven

Witkop, Catherine

Wood, Malissa

Wood, Susan

Woods, Nancy

Wright, Michelle

Wu, Horng-Shiuann

Wyman, Jean

Yano, Elizabeth

Yoles, Israel

Yong, Paul

Young Pierce, Jennifer

Young-Wolff, Kelly

Zahn, Christopher

Zakiniaeiz, Yasmin

Zamora-Ros, Raul

Zarkowski, Pamela

Zikmund-Fisher, Brian

Zimmerman, R. 